\title{
Presynaptic But Not Postsynaptic GABA Signaling at Unitary Mossy Fiber Synapses
}

\author{
Carolina Cabezas, ${ }^{1,2,3}$ Theano Irinopoulou, ${ }^{1,2,3}$ Grégory Gauvain, ${ }^{1,2,3}$ and Jean Christophe Poncer ${ }^{1,2,3}$ \\ ${ }^{1}$ INSERM, UMR 839, F75005, Paris, France, ${ }^{2}$ Université Pierre et Marie Curie, F75005, Paris, France, and ${ }^{3}$ Institut du Fer à Moulin, F75005, Paris, France
}

Dentate gyrus granule cells have been suggested to corelease GABA and glutamate both in juvenile animals and under pathological conditions in adults. Although mossy fiber terminals (MFTs) are known to express glutamic acid decarboxylase (GAD) in early postnatal development, the functional role of GABA synthesis in MFTs remains controversial, and direct evidence for synaptic GABA release from MFTs is missing. Here, using GAD67-GFP transgenic mice, we show that GAD67 is expressed only in a population of immature granule cells in juvenile animals. We demonstrate that GABA can be released from these cells and modulate mossy fiber excitability through activation of GABAB autoreceptors. However, unitary postsynaptic currents generated by individual, GAD67-expressing granule cells are purely glutamatergic in all postsynaptic cell types tested. Thus GAD67 expression does not endow dentate gyrus granule cells with a full GABAergic phenotype and GABA primarily instructs the pre- rather than the postsynaptic element.

\section{Introduction}

Dentate gyrus granule cells (GCs) are the main targets of cortical afferents to the hippocampal cortex. Their axons-the mossy fibers (MFs) - convey a complex signal to their postsynaptic targets comprising both monosynaptic, glutamatergic excitation and disynaptic, GABAergic inhibition (McBain, 2008). In addition to glutamate, mossy fiber terminals (MFTs) have been shown to contain GABA (Sandler and Smith, 1991), the GABAsynthesizing enzyme glutamate decarboxylase (GAD; Sloviter et al., 1996), and the vesicular transporter VIAAT (vesicular inhibitory amino acid transporter; Zander et al., 2010). Mixed (glutamatergic and GABAergic), apparently monosynaptic responses are recorded in CA3 pyramidal neurons upon stimulation of the granule cell layer, suggesting the two neurotransmitters might be coreleased from MFTs (Walker et al., 2001; Safiulina et al., 2006). This was observed both in juvenile [ $<$ P24 (postnatal day 24)] animals (Gutiérrez et al., 2003) and under pathological conditions in adults (Sloviter et al., 1996), suggesting MFs may be endowed with a developmental and activity-dependent switch of neurotransmitter modality.

However, the complex dentate gyrus circuitry complicates the interpretation of these data, which might reflect direct stimula-

Received 0ct. 27, 2011; revised June 30, 2012; accepted July 11, 2012.

Author contributions: C.C. and J.C.P. designed research; C.C. and T.I. performed research; G.G. contributed unpublished reagents/analytic tools; C.C., T.I., and J.C.P. analyzed data; C.C. and J.C.P. wrote the paper.

This work was supported by INSERM (Avenir Program to J.C.P.), the city of Paris, and Région lle-de-France (fellowship to C.C.). We thank Josh Huang and Tommaso Pizzorusso for kindly providing $\mathrm{G} 42$ mice, Dimitri Kullmann, Enrico Cherubini, Manuel Mameli, and Fiona Francis for critical reading of an earlier version of the manuscript, Richard Palmiter for the gift of anti-ZnT3 antibody, Charline Emmanuelli for preparing organotypic cultures, and Céline Dinocourt for expert advice.

Correspondence should be addressed to either Jean Christophe Poncer or Carolina Cabezas, INSERM UMR-S 839, 17 rue du Fer a Moulin, 75005 Paris, France, E-mail: jean-christophe.poncer@inserm.fr, or carolina.cabezas@icm-institute.org.

C. Cabezas' present address: ICM - Institut du Cerveau et de la Moelle épinière, 47 boulevard de I'Hôpital, F75013 Paris.

DOI:10.1523/JNEUROSCI.5543-11.2012

Copyright $\odot 2012$ the authors $\quad 0270-6474 / 12 / 3211835-06 \$ 15.00 / 0$ tion of local interneurons (Uchigashima et al., 2007). Paired recordings from individual GCs and postsynaptic pyramidal neurons (Mori et al., 2004) are needed to demonstrate activation of postsynaptic GABAA receptors at MF synapses. Here, we used a GAD67-EGFP mouse strain to show that GAD67 expression in MFTs is restricted to a subpopulation of immature GCs. Postsynaptic responses induced by direct stimulation of individual, GAD67-expressing GCs are purely glutamatergic. However, GABA can be released from these cells and activate GABAB receptors acting to control MF excitability.

\section{Materials and Methods}

Animals. G42 mice (Chattopadhyaya et al., 2004) were backcrossed on C57BL/ 6 background and only males were used for experiments. All the procedures performed conformed to the International Guidelines on the ethical use of animals.

Immunohistochemistry. P15 mice were anesthetized by i.p. injection of ketamine/xylazine and perfused transcardially with $4 \%(\mathrm{w} / \mathrm{v})$ paraformaldehyde in PBS. Brains were postfixed overnight and stored at $4^{\circ} \mathrm{C}$ in $30 \%$ sucrose for $48 \mathrm{~h}$. Transverse sections ( $40 \mu \mathrm{m}$ thick) were cut with a cryotome and stored at $-20^{\circ} \mathrm{C}$ in $30 \%$ ethylene glycol and $30 \%$ glycerol in PBS. For immunofluorescence, sections were preincubated $1 \mathrm{~h}$ with $0.3 \%$ Triton X-100 and 5\% goat serum in PBS and incubated overnight at $4^{\circ} \mathrm{C}$ with the primary antibodies: GFP (chicken, 1:1000, Millipore Bioscience Research Reagents); Prox1 (rabbit, 1:10,000, Millipore Bioscience Research Reagents); GAD67 (mouse, 1:2000, Millipore Bioscience Research Reagents); Calbindin D-28K (mouse, 1:1000, Swant); Doublecortin (rabbit, 1:2000; Cell Signaling Technology); NeuN (mouse, 1:250, Millipore Bioscience Research Reagents); ZnT3 (rabbit, 1:1000, kindly provided by R. Palmiter, University of Washington School of Medicine, Seattle, WA). Sections were rinsed in PBS, incubated $1 \mathrm{~h}$ with secondary antibodies (goat Cy3-coupled, 1:600; goat Cy5-coupled, 1:400; donkey-FITC, 1:500, Jackson Laboratory), rinsed in PBS and mounted with Mowiol/Dabco $(25 \mathrm{mg} / \mathrm{ml})$.

Acquisition and analysis of confocal images. Images from double- and triple-immunostainings were obtained by sequential confocal microscopy (Leica SP2 or SP5) using $40 \times / 1.25$ N.A and $63 \times / 1.32$ N.A objectives. Stacks of $10-40$ sections $(0.5 \mu \mathrm{m}$ apart $)$ were acquired with 
acquisition parameters kept constant. Confocal images were analyzed with Imaris (Bitplane) and MetaMorph (Molecular Devices) software. Cells were counted manually and were considered immunopositive for each marker when the fluorescence signal was larger than twice the background fluorescence. For quantification of MFTs, images were acquired with a $100 \times / 1.40$ N.A. objective and a $0.25 \mu \mathrm{m} z$-step. MFTs were defined as three-dimensional (3D) objects of volume $>3.14 \mu \mathrm{m}^{3}$ with colocalized immunofluorescence for ZnT3 and either GFP or calbindin. Their total number was computed and compared with the number of GAD67-immunopositive MFTs.

Electrophysiology. Horizontal hippocampal slices were prepared from postnatal day 10-20 G42 mice as described previously (Scheuber et al., 2004). For recording, slices were transferred to a submerged chamber maintained at $31^{\circ} \mathrm{C}$, mounted on an upright microscope (Olympus BX51WI) and superfused at $2.5 \mathrm{ml} / \mathrm{min}$ with ACSF composed of (in mM): $124 \mathrm{NaCl}, 26.2 \mathrm{NaHCO}_{3}, 11 \mathrm{D}$-glucose, $2.5 \mathrm{KCl}, 1 \mathrm{NaH}_{2} \mathrm{PO}_{4}, 2 \mathrm{CaCl}_{2}$ and $3 \mathrm{MgCl}_{2}$. Whole-cell recordings from GCs were made using borosilicate electrodes (3-5 $\mathrm{M} \Omega$ resistance) filled with (in $\mathrm{mm}$ ): $120 \mathrm{KMeSO}_{3}, 8 \mathrm{KCl}$, 10 HEPES, 5 EGTA, $3 \mathrm{MgCl}_{2}$ (pH 7.4, 290mOsm). Signals were filtered at $10 \mathrm{kHz}$ using a Multiclamp amplifier and digitized at $20 \mathrm{kHz}$ using Clampex software (Molecular Devices). All parameters were analyzed offline with Clampfit. Liquid junction potentials were computed using Clampex. No correction for seal resistance was used when estimating membrane resistance or resting membrane potential.

GABA release from MFs was evaluated from the probability of antidromic stimulation of GCs voltage-clamped at $-70 \mathrm{mV}$, using pipettes filled with (in mM): $120 \mathrm{~K}$-gluconate, $10 \mathrm{KCl}, 10 \mathrm{HEPES}, 0.1$ EGTA, 4 MgATP, 0.4 $\mathrm{Na}_{3}$ GTP. Recordings were made in the presence of bicuculline $(20 \mu \mathrm{M})$, DL-APV $(100 \mu \mathrm{M}), 2$,3-dihydroxy-6-nitro-7-sulfonyl-benzo[f] quinoxaline (NBQX; $10 \mu \mathrm{M}$ ), group II mGluR antagonist LY341495 (4 $\mu \mathrm{M})$ and A1 receptor antagonist DPCPX (100 nM). Antidromic stimulation was elicited at $0.2 \mathrm{~Hz}$ with a monopolar electrode placed in stratum (st.) lucidum (300-700 $\mu \mathrm{m}$ apart from st. granulosum). Trains of orthodromic action currents were elicited through the patch pipette, with sequences of depolarizinghyperpolarizing voltage pulses $(+70 \mathrm{mV}$ for $5 \mathrm{~ms}$ and at $-30 \mathrm{mV}$ for $15 \mathrm{~ms}$, 30 trials at $25 \mathrm{~Hz}$ ). Stimulation intensities and duration were typically $400-$ $850 \mu \mathrm{A}$ and $80-200 \mu \mathrm{s}$.

Analysis of unitary PSCs. Organotypic hippocampal slices from 7- to 8-dold G42 mice were prepared as described previously (Stoppini et al., 1991). Slices were kept in culture 10-12 d before recording to allow regrowth of severed axons. Whole-cell recordings from hilar interneurons, mossy cells or CA3 pyramidal cells were made with electrodes filled with (in mM): 115 $\mathrm{CsMeSO}_{4}, 20 \mathrm{CsCl}, 10 \mathrm{HEPES}, 10$ EGTA, $4 \mathrm{MgATP}$, and $0.4 \mathrm{Na}_{3} \mathrm{GTP}$.

Photostimulation of individual GCs was performed with $200 \mu \mathrm{M}$ MNIglutamate (4-methoxy-7- nitroindolinyl-caged $l$-glutamate; Tocris Bioscience) using a $405 \mathrm{~nm}$, laser diode beam (Deepstar, Omicron) conducted through a multimode fiber and alignment device (Prairie Technologies) set to generate $\mathrm{a}<3 \mu \mathrm{m}$ spot in the objective focal plane. In these conditions, moving the spot along the apical dendrite $>10 \mu \mathrm{m}$ away from the soma of a GC or onto immediately neighboring GCs, or defocusing by $10 \mu \mathrm{m}$ prevented reliable firing of GCs, as detected in cell attached recordings (see Fig. $2 B$ ). Single GCs were stimulated at $0.2 \mathrm{~Hz}$, while postsynaptic neurons were held at either $-70,-40$ or $0 \mathrm{mV}$ to distinguish GABAergic and glutamatergic responses based on reversal potential ( -48 and $+3 \mathrm{mV}$, respectively). Membrane potentials were not corrected for liquid junction potential $(+2.6$ $\mathrm{mV}$ as estimated using Clampex).

Statistical significance was assessed using Mann-Whitney or Wilcoxon tests using SigmaStat (SPSS).

\section{Results}

The GABAergic phenotype of hippocampal GCs has been described at early postnatal stages ( $<$ P24; Gutiérrez et al., 2003), when most GCs still undergo differentiation and maturation (Altman and Bayer, 1990). We thus asked whether GAD expression was homogeneous in juvenile mouse GCs. G42 mice express EGFP under the control of $\sim 10 \mathrm{~kb}$ of Gad1 promoter. In neocortex, EGFP expression is restricted to parvalbumin interneurons (Chattopadhyaya et al., 2004). In contrast, in the dentate gyrus,
EGFP + cells are mostly GCs, as evidenced by $99.8 \%$ colocalization with Prox-1 immunostaining (Fig. 1A). These neurons extend ramified dendrites in st. moleculare and project to st. lucidum of area CA3 (Fig. $1 \mathrm{~B}$ ). However, their phenotype is typical of immature but not newborn GCs, with expression of doublecortin but not calbindin (Fig. 1C), low membrane capacitance $(50.7 \pm 7.6$ vs $99.5 \pm 18.0 \mathrm{pF}$ for EGFP - cells, $p<0.05)$ and moderate input resistance $(841 \pm 77$ vs $879 \pm 74 \mathrm{M} \Omega$ for EGFPcells, $n=20$ and 33, $p=1.0$ ).

We confirmed that EGFP reflects GAD67 expression in triple immunostaining experiments followed by confocal imaging and $3 \mathrm{D}$ reconstructions (Fig. $1 D$ ). At P15, $\approx 75 \%$ of MFTs identified by expression of EGFP and the zinc transporter ZnT3 were also immunopositive for GAD67. In contrast, $<10 \%$ of mature MFTs immunopositive for calbindin and ZnT3 also showed GAD67 expression (Fig. 1D3). Thus, in juvenile animals, GAD67 is expressed in a subpopulation of MFTs originating from immature GCs and G42 mice permit their specific identification.

Newborn GCs fire small-amplitude action potentials which may be unable to evoke transmitter release (Overstreet-Wadiche et al., 2006). We therefore examined the firing properties of GAD67expressing GCs in juvenile G42 mice. Cells were recorded in currentclamp mode and held at their resting potential, which was similar in EGFP + vs EGFP - cells $(-68.1 \pm 3.1$ and $-67.1 \pm 2.7 \mathrm{mV}, n=17$ and 32, $p=1.0$; Fig. 2 A). A 100 pA depolarizing current pulse elicited overshooting action potentials in 100\% EGFP - but only $55 \%$ EGFP+ GCs. In addition, action potentials were smaller in amplitude in EGFP+ compared with EGFP - cells (36.8 \pm 3.7 vs $49.0 \pm$ $3.1 \mathrm{mV}$ from threshold, $p<0.05$; Fig. $2 A, B)$.

GC heterogeneity with respect to GAD67 expression (Fig. 1), together with the presence of local interneurons in the granule cell layer and hilus (Lübke et al., 1998; Houser, 2007) complicates the interpretation of postsynaptic responses evoked by extracellular electrical stimulation (Walker et al., 2001; Safiulina et al., 2006; Uchigashima et al., 2007). We examined the postsynaptic responses evoked by photostimulation of individual, visually identified GCs using local photolysis of MNI-glutamate in hippocampal organotypic slices from G42 mice. Laser pulse power and duration were set in each experiment just suprathreshold to reliably elicit single action potentials in the targeted GC but not in neighboring cells or dendrites, as detected from cell-attached recordings (see Materials and Methods; Fig. 2B). When GCs were stimulated at $0.2 \mathrm{~Hz}$, mean peak latency of action potentials ranged 3.1-8.5 ms $(5.1 \pm 0.4, n=18)$ from laser pulse onset, with a mean coefficient of variation (CV) of $0.12 \pm 0.02$ (Fig. 2D). Whereas EGFP - cells could be made to fire up to $25 \mathrm{~Hz}$ using photostimulation, firing of EGFP+GCs was reliable up to $2 \mathrm{~Hz}$ stimulation but abruptly declined at higher frequencies $(n=5-7$, Fig. 2C). Unitary PSCs (uPSCs) were tested in hilar and CA3 neurons. The overall probability of connected cell pairs was $6.0 \%$ ( $n=39$ of 648). In two recordings from CA3 pyramidal cells, the mGluR agonist L-AP4 $(20 \mu \mathrm{M})$ reduced uPSC amplitude by $74.9 \%$, typical of responses originating from MFTs (McBain, 2008).

We assessed the nature of uPSCs based on their reversal potential ( $+3 \mathrm{mV}$ and $-48 \mathrm{mV}$ for AMPA/kainate- and GABAAreceptor-mediated currents, respectively). uPSCs recorded from 13 of 14 hilar interneurons or mossy cells upon stimulation of either EGFP $+(n=11)$ or EGFP $-(n=2)$ GCs were entirely mediated by glutamate receptors, reversing around $0 \mathrm{mV}$. The amplitude of unitary EPSCs (uEPSCs) recorded in hilar interneurons upon stimulation of EGFP+ GCs was $30.9 \pm 4.5 \mathrm{pA}(n=8)$. Contribution of disynaptic inhibition to uPSCs in CA3 pyrami- 

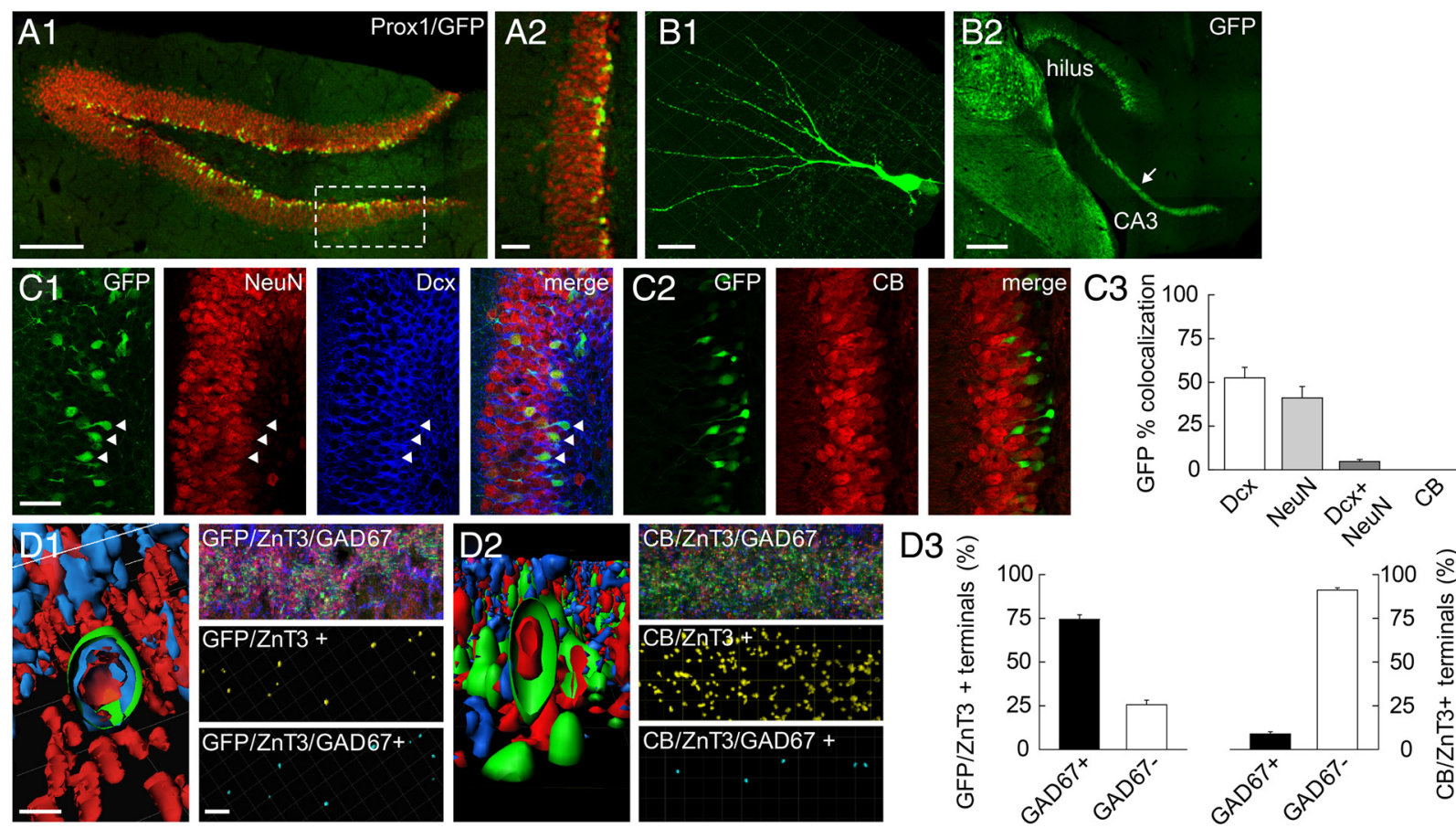

Figure 1. GAD67 is expressed only in immature GCs in juvenile dentate gyrus. A1, Maximum projection of confocal images of double Prox1(red)/EGFP(green) immunostaining in P15 hippocampal slices showing EGFP expression is restricted to Prox 1 + cells. A2, Enlarged view of boxed area in A1. B1, 3D reconstruction of an EGFP + GC. B2, EGFP immunostaining revealing MFs in st. lucidum of area CA3 (arrow). C1, Triple immunostaining of EGFP (green), NeuN (red), and doublecortin (Dcx; blue). Arrowheads, Colocalization of EGFP and Dcx. C2, Double immunostaining of EGFP(green) and calbindin(CB, red) showing no colocalization in GCs. C3, Proportions of EGFP + GCs immunopositive for Dcx, NeuN or CB. $n=12$ slices from 5 P15 animals. D1, Left, 3D reconstruction of individual EGFP + (green) MFTs showing colocalization with ZnT3 (red) and GAD67 (blue). Right, MFTs identified with dual GFP/ZnT3 (yellow dots) or triple GFP/ZnT3/GAD67 (blue) colocalization after 3D reconstruction. D2, same as D1 for calbindin (green)/ZnT3 (red)/GAD67 (blue) triple immunostaining. D3, Quantification of GAD67 colocalization in GFP/ZnT3 + (left, $n=15$ slices/5 animals) and CB/ZnT3(right) MFTs ( $n=7$ slices/3 animals). Scale bars (in $\mu \mathrm{m}$ ): $\mathbf{A 1}, 200 ; A 2,50 ; \mathbf{B 1}, 10 ; \mathbf{B 2}, 200 ; \mathbf{C 1}, \mathbf{C 2}, 40 ; \mathbf{D 1}, \mathbf{D 2}$, left, 2; D1, D2, right, 10.

dal cells may prevent unambiguous detection of monosynaptic responses (Mori et al., 2004). It was therefore minimized in all recordings by philanthotoxin $(5 \mu \mathrm{M})$ which partially blocked evoked EPSC in st. lucidum and hilar interneurons $(-58.7 \pm$ $6.6 \%$ of control, $n=6, p<0.05)$. In these conditions, photostimulation of EGFP - GCs evoked uPSCs with no outward component at $0 \mathrm{mV}$ in all four pyramidal cells tested, indicating they were entirely glutamatergic (Fig. 2E,F). Similarly, uPSCs induced by stimulation of EGFP+ GCs were purely glutamatergic in eight of 13 tested pairs (Fig. $2 G, H$ ). The mean uEPSC latency $(15.6 \pm 2.2 \mathrm{vs} 10.7 \pm 2.2 \mathrm{~ms}$ from laser onset, $p=0.2)$ and its CV $(0.15 \pm 0.02$ vs $0.10 \pm 0.04, p=0.2)$ were not significantly different for uEPSCs evoked from EGFP + vs EGFP- GCs, but their amplitude was different $(33.6 \pm 3.4$ vs $58.8 \pm 10.4 \mathrm{pA}, p<$ $0.05)$. In the five remaining recordings, outward currents were sometimes observed at -40 and $0 \mathrm{mV}$ (Fig. $2 J, K$ ). However, the onset latency of these currents was significantly larger than that of inward currents $(+68.1 \pm 15.0 \%, p<0.02)$ as was the CV of their latency $(0.32 \pm 0.03$ vs $0.17 \pm 0.04, p<0.05$; Fig. $2 L)$, suggesting those outward currents were disynaptic IPSCs. Accordingly, focal application of NBQX (1 mM) onto the apical dendrites of postsynaptic pyramidal cells suppressed (by $99.3 \pm 0.4 \%$ of control amplitude) the uPSC induced by photostimulation of GAD67+ GCs in three tested pairs (two with a monosynaptic EPSC and one with a monosynaptic EPSC followed by a delayed, disynaptic IPSC, Fig. $2 M, N)$. We conclude that phasic firing in GCs expressing GAD67 fails to directly activate postsynaptic GABAA receptors in CA3 pyramidal cells from juvenile mouse hippocampus.

We next asked whether GABA was released from GAD67+ GCs. Presynaptic GABA receptors have been shown to influence
MF excitability and transmitter release (Vogt and Nicoll, 1999; Ruiz et al., 2003) and may thus be used to probe GABA release from MFs. Antidromic action currents were evoked in GCs by extracellular stimulation in st. lucidum. In the presence of GABAA, adenosine $\mathrm{Al}$ and both ionotropic and group II metabotropic glutamate receptor antagonists (see Materials and Methods), a $25 \mathrm{~Hz}$ train of 30 action currents evoked orthodromically by somatic current pulses had no significant effect on antidromic stimulation in EGFP- GCs $(+6.2 \pm 16.6 \%, n=7$, $p=0.7$; Fig. $3 A, B)$. However, the same train of orthodromic action potentials induced a transient (recovery time constant $\sim 30$ s) reduction in probability of antidromic stimulation by $52.4 \pm 8.4 \%$ in EGFP+ GCs $(n=7, p<0.001$; Fig. $3 C, D)$. This effect was abolished by the GABAB-receptor antagonist CGP55845 ( $3 \mu \mathrm{M} ;-12.4 \pm 6.6 \%$ of control, $n=5, p=0.2$; Fig. $3 C, D)$, indicating $G A B A B$ receptors were activated upon GABA release and modulate axonal excitability in GAD67+ GCs. Both CGP55845 and the high-affinity GIRK channel antagonist tertiapin-Q (50 nM) facilitated antidromic stimulation in basal conditions (Fig. $3 C-F$ ), suggesting GABAB receptors and GIRK channels may be activated at rest. In 4 of 5 experiments, stimulation charge was therefore reduced (by $9.7 \pm 1.8 \%$ ) after application of either antagonist to prevent supramaximal stimulation. In these conditions, tertiapin-Q prevented the effect of a train of orthodromic action currents on antidromic stimulation in EGFP+ GCs $(-2.1 \pm 5.7 \%$ of control, $p=0.6, n=5$; Fig. $3 E, F)$. Together, these results demonstrate that GABA is released from GAD67+ GCs upon repetitive firing and activates GABAB receptors likely acting on GIRK channels to reduce MF excitability. 
A

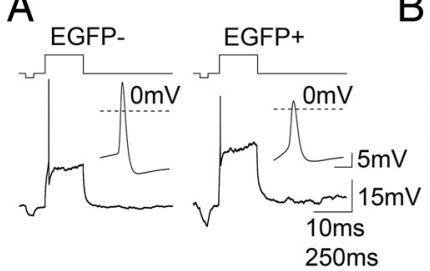

B
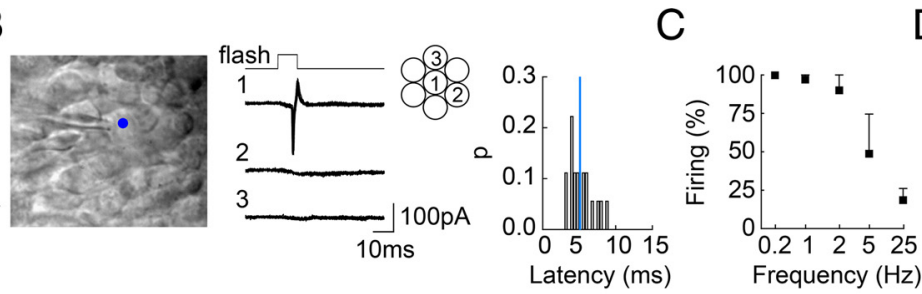

D

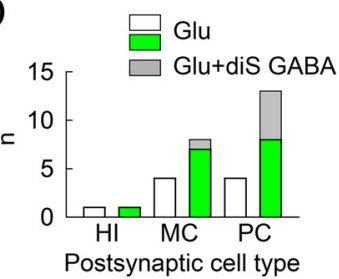

$\mathrm{F}$
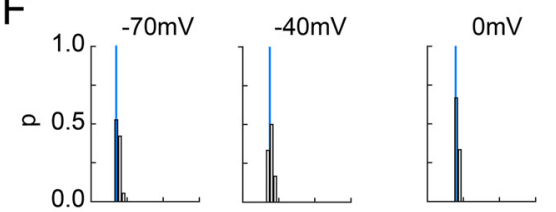

$\mathrm{H}$
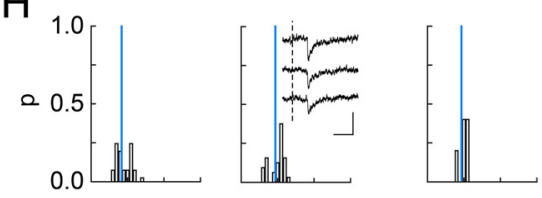

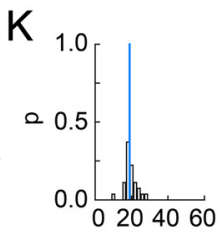
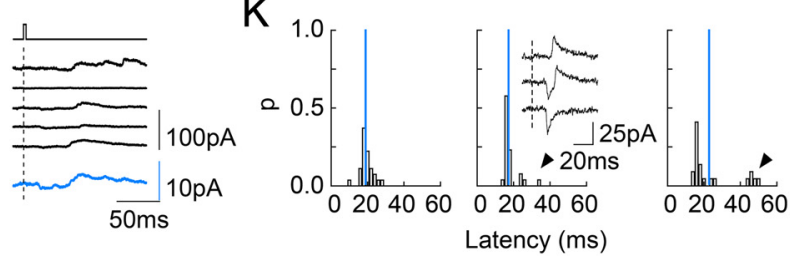

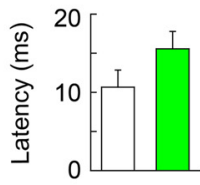

$M$
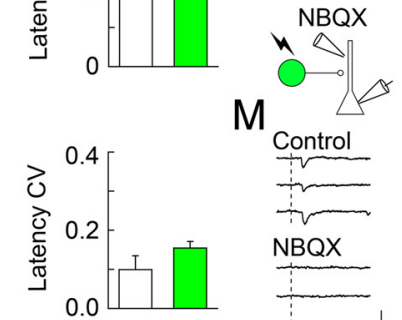

Control

$\underbrace{\text { Control }}_{+r}$

Trum

NBQX
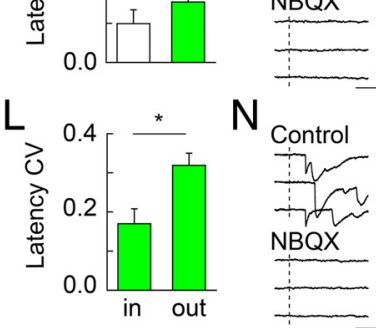

: $100 \mathrm{pA}$

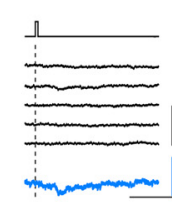

$J$

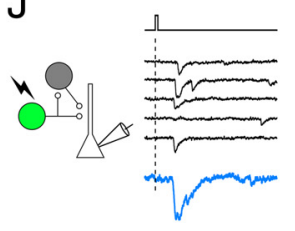

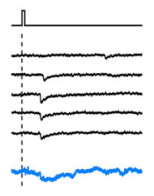

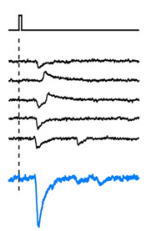

Figure 2. Properties of unitary postsynaptic responses from individual GCs. A, Action potentials evoked in EGFP - (left) and EGFP + (right) GCs by a 100 pA depolarizing pulse from rest. Insets, Detail of action potentials. $B$, Left, GC from organotypic slice culture recorded in cell-attach mode with a $405 \mathrm{~nm}$ light spot ( $\emptyset 3-5 \mu \mathrm{m})$ focused on cell soma. Middle, Action currents recorded from neuron 1 in cell-attached mode upon 5 ms laser pulse focused on its soma (1) but not on immediately neighboring neurons ( 2 and 3). Twenty consecutive traces each. Right, Distribution of mean latencies from onset of laser pulse to action potential peak, from 18 recordings as in B. P, Proportion. Blue bar, Mean latency. $\boldsymbol{C}$, Reliability of firing upon photostimulation of EGFP $+\mathrm{GCS}$ at various frequencies estimated from trains of 15 pulses $(n=5-7)$. D. Characteristics of uPSCs recorded in various postsynaptic cells. HI, Hilar interneurons; MC, mossy cells; PC, CA3 pyramidal cells. $n$, Number of observations of pure glutamatergic (white and green bars for EGFP - and EGFP + GCs, respectively) and mixed glutamatergic and disynaptic GABAergic currents (gray bars) for each cell type. $\boldsymbol{E}$, Example of uPSC recorded at various holding potentials from a CA3 pyramidal cell upon stimulation of an EGFP - GC. Blue traces, Average of $20-50$ responses. $F$, Corresponding latency distributions of uPSCS showing no change of the mean (blue bar) at different potentials. $\boldsymbol{G}, \boldsymbol{H}$, Same as in $\boldsymbol{E}$ and $\boldsymbol{F}$ for a pure glutamatergic uPSC recorded from a $C A 3$ pyramidal cell upon stimulation of an EGFP+ GC. Inset, Sample traces showing uPSCs at $-40 \mathrm{mV}$. I, Synaptic latencies (top) and their CV (bottom) measured from laser pulse onset to uPSC onset upon photostimulation of EGFP - (white, $n=$ 4) and EGFP + (green, $n=7$ ) GCs. J, $\boldsymbol{K}$, Same as $\mathbf{G}$ and $\boldsymbol{H}$ for a mixed, glutamatergic, and disynaptic GABAergic uPSC. Note reversal of GABA component at $+40 \mathrm{mV}$ and later onset compared with inward, glutamatergic component (onset, and arrowheads in latency distributions). L, Compared CV of synaptic latencies for inward (IN, - $70 \mathrm{mV}$ ) and outward (OUT, $0 \mathrm{mV}$ ) uPSCs evoked upon stimulation of the same EGFP $+\mathrm{GCs}(n=3, p<0.01) . \boldsymbol{M}, \mathbf{N}$, Examples of uPSCs recorded at $-70 \mathrm{mV}$ from CA3 pyramidal cells before (control) and after focal application of $1 \mathrm{~mm}$ NBQX onto their apical dendrite. Note that both monosynaptic uEPSCS $(\boldsymbol{M}, \boldsymbol{N})$ and disynaptic IPSCS $(\boldsymbol{N})$ are totally suppressed by NBQX.

\section{Discussion}

Our results challenge the controversial hypothesis that dentate gyrus GCs in juvenile hippocampus may convey fast, monosynaptic inhibition to their postsynaptic targets. We have shown that, although GABA can be released from some GCs and act to modulate MF excitability, it fails to induce detectable monosynaptic, unitary IPSCs in various GC postsynaptic targets in hippocampal organotypic slice cultures.

Dual glutamate/GABA transmission at MF synapses is supported by the following evidence: (1) GAD and VIAAT are expressed in MFTs (Sloviter et al., 1996; Gutiérrez et al., 2003; Zander et al., 2010), (2) GABA is released from isolated MF synaptosomes (Gómez-Lira et al., 2002), (3) GABAA receptors are expressed in the postsynaptic density facing GAD-immunopositive MFTs (Bergersen et al., 2003), and (4) IPSCs are detected upon extracellular stimulation in st. granulosum (Walker et al., 2001; Gutiérrez et al., 2003; Safiulina et al., 2006; but see Mori et al., 2004; Uchigashima et al., 2007; Toni et al., 2008). Our results appear consistent with some of these observations but do not support the conclusion that postsynaptic GABAA receptors are activated by GABA release at MFTs upon phasic activity.
We show GAD67 is expressed mostly in a population of relatively immature dentate gyrus GCs with input resistance intermediate between newborn and mature GCs. This observation is consistent with a recent report using another GAD67-GFP mouse strain (Zhao et al., 2010). Although a majority of MFTs already express calbindin and not GAD67, a significant proportion of GCs still undergo differentiation and maturation after birth in rodents (Altman and Bayer, 1990). Therefore our observation likely accounts for the reported timing of GAD67 expression during the first 3 postnatal weeks (Gutiérrez et al., 2003). Thus, GAD67 expression in GCs does not reflect a specific ontogenetic stage but instead represents a new intrinsic marker of GC differentiation.

We report a GABAB receptor-mediated modulation of MF excitability. This represents the first evidence at single cell level for GABA release from MFs. The mechanism of GABA release from GAD67 + MFs remains to be fully elucidated and may be either synaptic (Gutiérrez et al., 2003) or involve the reverse action of the GAT1 transporter upon repetitive firing (Wu et al., 2007). Repetitive firing in a GAD67 + GC transiently reduced the probability of evoked antidromic spikes. This effect was observed with saturating concentrations of glutamate receptor antagonists 
A

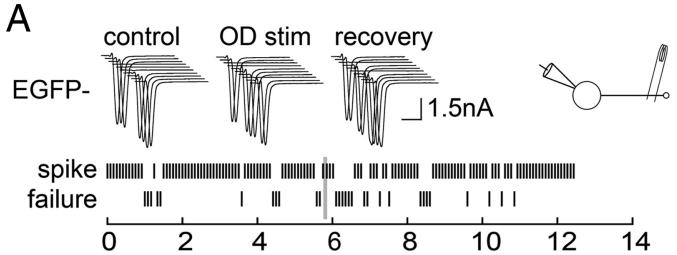

C

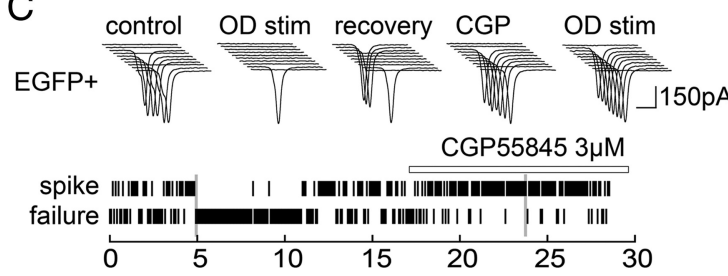

$E$

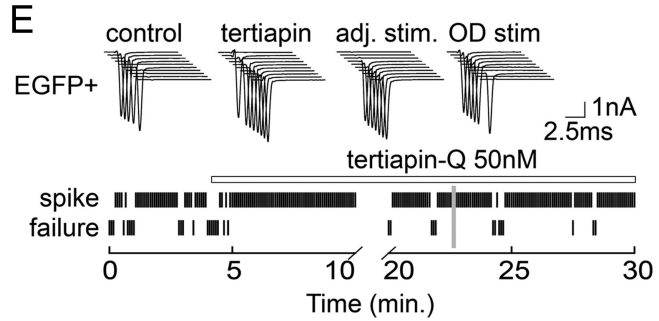

$\mathrm{B}$

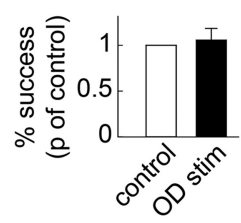

D

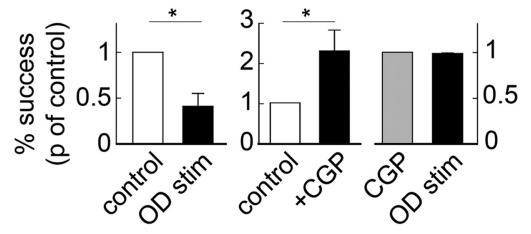

$\mathrm{F}$

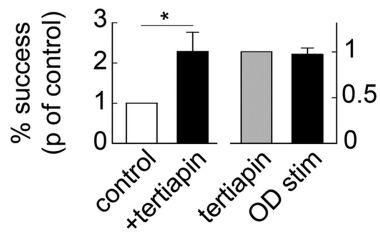

Figure 3. Direct evidence for GABA release at MFTs from GAD67-expressing GCs. $A$, Top, 10 consecutive responses of an EGFP- GC from acute hippocampal slice to antidromic stimulation, before and after 30 orthodromic stimulations at $25 \mathrm{~Hz}$ (0D stim, gray bar). Bottom, Raster plots of antidromic responses. B, D, Summary data from 7 EGFP - and 7 EGFP + cells ( $n=5$ for CGP55845 data). Data are normalized to success rate before OD stim. $\boldsymbol{C}, \boldsymbol{E}$, Same as $\boldsymbol{A}$ in EGFP $+\mathrm{GCs}$. Application of the GABAB receptor antagonist CGP55845 or the high-affinity GIRK channel antagonist tertiapin- $Q$ facilitated antidromic stimulation. After readjusting stimulation intensity to reach $<90 \%$ of success ( $71 \pm 12 \%$ on average), orthodromic stimulation failed to affect antidromic stimulation. $\boldsymbol{F}$, Summary data from 7 (effect of tertiapin- $Q$ ) and 5 (OD stim. in the presence of tertiapin- 0 ) EGFP + cells. ${ }^{*} p<0.05$.

and is therefore unlikely to reflect disynaptic inhibition. Given the distance of antidromic stimulation from GC layer (300-700 $\mu \mathrm{m}$ ), it also seems unlikely to reflect changes in somatic excitability. Its sensitivity to both CGP55845 and tertiapin-Q suggests instead that GABA released along the axon of the recorded GC activates GABAB autoreceptors coupled to GIRK channels. Although coupling of presynaptic $\mathrm{GABAB}$ receptors to transmitter release may involve modulation of $\mathrm{Ca}^{2+}$ rather than $\mathrm{K}^{+}$channels (Lüscher et al., 1997), several recent reports reveal the presence of presynaptic GIRK channels (Kulik et al., 2006; Ladera et al., 2008; Michaeli and Yaka, 2010) that may be coupled to GABAB receptors (Kulik et al., 2006; Lujan et al., 2009). The mechanisms by which activation of GIRK channels may induce prolonged $(>30$ s) changes in axonal excitability remain to be fully characterized but may involve hyperpolarization-induced, deinactivation of potassium currents. Such mechanism has been suggested to account for the effect of presynaptic GABAA receptor activation on MF excitability, which also largely outlasts the GABA current itself (Ruiz et al., 2003). Although we did not specifically test for the possible recruitment of presynaptic GABAA receptors in this study, it may be limited in GAD67+ cells due to their weak expression of the $\delta$ subunit (our unpublished observations) which likely contributes to GABAA receptor-mediated modulation of MF excitability (Ruiz et al., 2010).

Direct stimulation of individual, GAD67 + GCs fails to elicit monosynaptic, unitary IPSCs in postsynaptic neurons. The onset latency of uPSCs from GAD67+ GCs was slightly longer than that of uPSCs evoked from GAD67- GCs, possibly reflecting reduced axonal conduction velocity in these rather immature neurons (Bucher and Goaillard, 2011). Nevertheless, the monosynaptic nature of uPSCs was attested by (1) their short latency (10.7 and $15.6 \mathrm{~ms}$ from laser onset, for GAD67- and GAD67+
GCs respectively, from which the 3.1-8.5 ms firing latency of GCs should be subtracted) and (2) the very low CV of their latency ( 0.10 and 0.15 , respectively), less than that of unitary EPSCs evoked by direct stimulation of individual GCs ( 0.19 ; Jonas et al., 1993). In a few recordings, a GABAergic component was detected with a delay of 5-18 ms compared with the monosynaptic glutamatergic component. This delay is compatible with that observed for disynaptic inhibition in CA3 pyramidal neurons (Mori et al., 2004). This conclusion is further substantiated by the suppression of this GABAergic component by focal application of NBQX onto pyramidal cell apical dendrites. Disynaptic IPSCs were observed despite the presence of philanthotoxin, suggesting that excitatory synapses onto hilar and/or CA3 interneurons were not entirely blocked by this antagonist of GluA2-lacking AMPARs (McBain, 2008). Consistent with previous results using paired recordings from unidentified GCs (Mori et al., 2004), our results suggest GAD67 + GCs do not evoke monosynaptic IPSCs in their postsynaptic targets upon phasic firing. We cannot exclude that some postsynaptic GABAA receptors may be activated by GABA released from GAD67 + MFTs (Bergersen et al., 2003). However, such receptors may not be present at a high enough density or proximity to the active zone to generate a detectable synaptic response, even in the presence of NBQX. Thus, although our observations were made in organotypic cultures, we suggest the apparent monosynaptic IPSCs recorded in CA3 pyramidal neurons upon st. granulosum stimulation in acute hippocampal slices (Walker et al., 2001; Gutiérrez et al., 2003; Safiulina et al., 2006) likely originate from direct stimulation of the profuse hilar interneuron processes in this layer (Lübke et al., 1998). In these studies, the putative MF origin of these IPSCs was based on their sensitivity to presynaptic mGluR agonists and use-dependent facilitation, which are also observed at synapses from some in- 
terneurons onto CA3 pyramidal cells (Poncer et al., 2000). Paired recordings from hilar/CA3 interneurons and CA3 pyramidal cells may help resolve this issue.

If not to convey fast, monosynaptic inhibition to their targets, what might be the functional role of GABA synthesis in MFTs from immature GCs? Our experiments demonstrate that GABA can be released from GAD67+ GCs and activate autoreceptors. Therefore the primary function of GABA released from GCs may be to instruct the pre- rather than the postsynaptic element. The functional impact of such feedback action may depend on the subtypes of autoreceptors that are activated. Both GABAA and GABABB receptors are expressed at MFTs. In addition to modulating axonal excitability (Ruiz et al., 2003; our results) and transmitter release (Vogt and Nicoll, 1999) activation of presynaptic GABAA and GABAB receptors may also serve to modulate growth cone guidance (Xiang et al., 2002; Bouzigues et al., 2007). This latter effect might be critical for the process of MF elongation which strikingly coincides with GAD67 expression in MFTs (Faulkner et al., 2008).

\section{References}

Altman J, Bayer SA (1990) Migration and distribution of two populations of hippocampal granule cell precursors during the perinatal and postnatal periods. J Comp Neurol 301:365-381.

Bergersen L, Ruiz A, Bjaalie JG, Kullmann DM, Gundersen V (2003) GABA and GABAA receptors at hippocampal mossy fibre synapses. Eur J Neurosci 18:931-941.

Bouzigues C, Morel M, Triller A, Dahan M (2007) Asymmetric redistribution of GABA receptors during GABA gradient sensing by nerve growth cones analyzed by single quantum dot imaging. Proc Natl Acad Sci U S A 104:11251-11256.

Bucher D, Goaillard JM (2011) Beyond faithful conduction: short-term dynamics, neuromodulation, and long-term regulation of spike propagation in the axon. Prog Neurobiol 94:307-346.

Chattopadhyaya B, Di Cristo G, Higashiyama H, Knott GW, Kuhlman SJ, Welker E, Huang ZJ (2004) Experience and activity-dependent maturation of perisomatic GABAergic innervation in primary visual cortex during a postnatal critical period. J Neurosci 24:9598-9611.

Faulkner RL, Jang MH, Liu XB, Duan X, Sailor KA, Kim JY, Ge S, Jones EG, Ming GL, Song H, Cheng HJ (2008) Development of hippocampal mossy fiber synaptic outputs by new neurons in the adult brain. Proc Natl Acad Sci U S A 105:14157-14162.

Gómez-Lira G, Trillo E, Ramírez M, Asai M, Sitges M, Gutiérrez R (2002) The expression of GABA in mossy fiber synaptosomes coincides with the seizure-induced expression of GABAergic transmission in the mossy fiber synapse. Exp Neurol 177:276-283.

Gutiérrez R, Romo-Parra H, Maqueda J, Vivar C, Ramìrez M, Morales MA, Lamas M (2003) Plasticity of the GABAergic phenotype of the "glutamatergic" granule cells of the rat dentate gyrus. J Neurosci 23:5594-5598.

Houser CR (2007) Interneurons of the dentate gyrus: an overview of cell types, terminal fields and neurochemical identity. Prog Brain Res 163:217-232.

Jonas P, Major G, Sakmann B (1993) Quantal components of unitary EPSCs at the mossy fibre synapse on CA3 pyramidal cells of rat hippocampus. J Physiol 472:615-663.

Kulik A, Vida I, Fukazawa Y, Guetg N, Kasugai Y, Marker CL, Rigato F, Bettler B, Wickman K, Frotscher M, Shigemoto R (2006) Compartmentdependent colocalization of Kir3.2-containing $\mathrm{K}+$ channels and GABAB receptors in hippocampal pyramidal cells. J Neurosci 26:4289-4297.

Ladera C, del Carmen Godino M, José Cabañero M, Torres M, Watanabe M, Luján R, Sánchez-Prieto J (2008) Pre-synaptic GABA receptors inhibit glutamate release through GIRK channels in rat cerebral cortex. J Neurochem 107:1506-1517.

Lübke J, Frotscher M, Spruston N (1998) Specialized electrophysiological properties of anatomically identified neurons in the hilar region of the rat fascia dentata. J Neurophysiol 79:1518-1534.
Lujan R, Maylie J, Adelman JP (2009) New sites of action for GIRK and SK channels. Nat Rev 10:475-480.

Lüscher C, Jan LY, Stoffel M, Malenka RC, Nicoll RA (1997) G proteincoupled inwardly rectifying $\mathrm{K}+$ channels (GIRKs) mediate postsynaptic but not presynaptic transmitter actions in hippocampal neurons. Neuron 19:687-695.

McBain CJ (2008) Differential mechanisms of transmission and plasticity at mossy fiber synapses. Prog Brain Res 169:225-240.

Michaeli A, Yaka R (2010) Dopamine inhibits GABA(A) currents in ventral tegmental area dopamine neurons via activation of presynaptic G-protein coupled inwardly-rectifying potassium channels. Neuroscience 165:1159-1169.

Mori M, Abegg MH, Gähwiler BH, Gerber U (2004) A frequencydependent switch from inhibition to excitation in a hippocampal unitary circuit. Nature 431:453-456.

Overstreet-Wadiche LS, Bensen AL, Westbrook GL (2006) Delayed development of adult-generated granule cells in dentate gyrus. J Neurosci 26:2326-2334.

Poncer JC, McKinney RA, Gahwiler BH, Thompson SM (2000) Differential control of GABA release at synapses from distinct interneurons in rat hippocampus. J Physiol 528:123-130.

Ruiz A, Fabian-Fine R, Scott R, Walker MC, Rusakov DA, Kullmann DM (2003) GABAA receptors at hippocampal mossy fibers. Neuron 39:961-973.

Ruiz A, Campanac E, Scott RS, Rusakov DA, Kullmann DM (2010) Presynaptic GABAA receptors enhance transmission and LTP induction at hippocampal mossy fiber synapses. Nat Neurosci 13:431-438.

Safiulina VF, Fattorini G, Conti F, Cherubini E (2006) GABAergic signaling at mossy fiber synapses in neonatal rat hippocampus. J Neurosci 26:597-608.

Sandler R, Smith AD (1991) Coexistence of GABA and glutamate in mossy fiber terminals of the primate hippocampus: an ultrastructural study. J Comp Neurol 303:177-192.

Scheuber A, Miles R, Poncer JC (2004) Presynaptic Cav2.1 and Cav2.2 differentially influence release dynamics at hippocampal excitatory synapses. J Neurosci 24:10402-10409.

Sloviter RS, Dichter MA, Rachinsky TL, Dean E, Goodman JH, Sollas AL, Martin DL (1996) Basal expression and induction of glutamate decarboxylase and GABA in excitatory granule cells of the rat and monkey hippocampal dentate gyrus. J Comp Neurol 373:593-618.

Stoppini L, Buchs PA, Muller D (1991) A simple method for organotypic cultures of nervous tissue. J Neurosci Methods 37:173-182.

Toni N, Laplagne DA, Zhao C, Lombardi G, Ribak CE, Gage FH, Schinder AF (2008) Neurons born in the adult dentate gyrus form functional synapses with target cells. Nat Neurosci 11:901-907.

Uchigashima M, Fukaya M, Watanabe M, Kamiya H (2007) Evidence against GABA release from glutamatergic mossy fiber terminals in the developing hippocampus. J Neurosci 27:8088-8100.

Vogt KE, Nicoll RA (1999) Glutamate and gamma-aminobutyric acid mediate a heterosynaptic depression at mossy fiber synapses in the hippocampus. Proc Natl Acad Sci U S A 96:1118-1122.

Walker MC, Ruiz A, Kullmann DM (2001) Monosynaptic GABAergic signaling from dentate to CA3 with a pharmacological and physiological profile typical of mossy fiber synapses. Neuron 29:703-715.

Wu Y, Wang W, Díez-Sampedro A, Richerson GB (2007) Nonvesicular inhibitory neurotransmission via reversal of the GABA transporter GAT-1. Neuron 56:851-865.

Xiang Y, Li Y, Zhang Z, Cui K, Wang S, Yuan XB, Wu CP, Poo MM, Duan S (2002) Nerve growth cone guidance mediated by G protein-coupled receptors. Nat Neurosci 5:843-848.

Zander JF, Münster-Wandowski A, Brunk I, Pahner I, Gómez-Lira G, Heinemann U, Gutiérrez R, Laube G, Ahnert-Hilger G (2010) Synaptic and vesicular coexistence of VGLUT and VGAT in selected excitatory and inhibitory synapses. J Neurosci 30:7634-7645.

Zhao S, Zhou Y, Gross J, Miao P, Qiu L, Wang D, Chen Q, Feng G (2010) Fluorescent labeling of newborn dentate granule cells in GAD67-GFP transgenic mice: a genetic tool for the study of adult neurogenesis. PloS One 5:e12506. 